\title{
Motivos y barreras para la práctica de actividad física percibidas por jugadores de goalball con discapacidad visual
}

\section{Perceived reasons and barriers for practicing physical activity by goalball players with visual disabilities}

Aldo Borja Avalos Solitario ${ }^{1}$; Aitor Iturricastillo Urteaga ${ }^{2}$; Daniel Castillo Alvira ${ }^{3}$; Estibaliz Romaratezabala Aldasoro ${ }^{2}$; Josune Rodríguez Negro ${ }^{1}$; Irati Aritzeta de Pérdigo ${ }^{1}$; Javier Yanci Irigoyen $^{2}$.

${ }^{1}$ Departamento de Educación Física y Deportiva, Facultad de Educación y Deporte, Universidad del País Vasco, UPV/EHU, Vitoria-Gasteiz, España.

${ }^{2}$ Society, Sports and Physical Exercise Research Group (GIKAFIT). Departamento de Educación Física y Deportiva, Facultad de Educación y Deporte, Universidad del País Vasco, UPV/EHU, Vitoria-Gasteiz, España.

${ }^{3}$ Facultad de las Ciencias de la Salud, Universidad Isabel I, Burgos, España. Contacto: estibaliz.romaratezabala@ehu.eus 


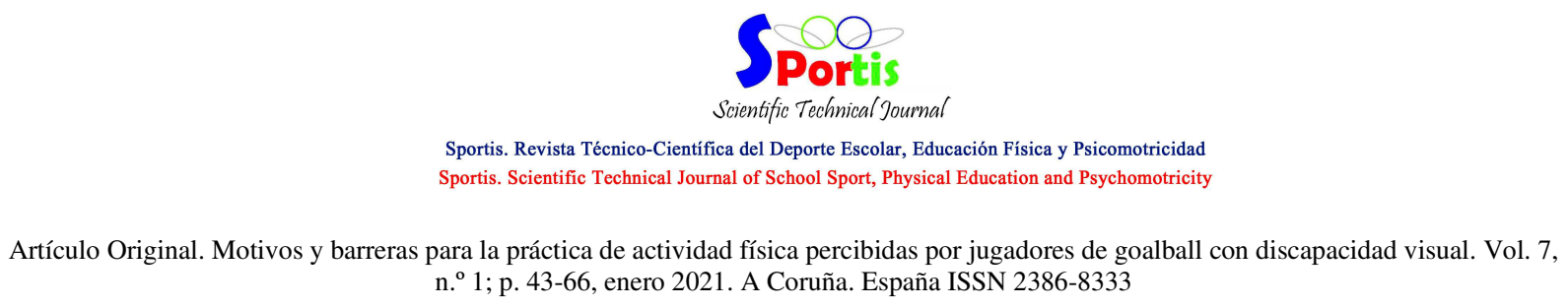

\title{
Resumen
}

El presente estudio tiene por objetivo analizar los motivos y las barreras para la práctica de actividad física (AF) en personas con discapacidad visual practicantes de goalball. En este estudio participaron 25 personas adultas $(32,7 \pm 11,9$ años), de las cuales 14 eran mujeres (31,3 $\pm 12,0$ años) y 11 eran hombres $(34,4 \pm 12,2$ años) teniendo todos licencia federativa en vigor expedida por la Federación Española de Deportes para Ciegos. Los participantes completaron el cuestionario Motivos y Barreras para la Actividad Física y el Deporte $(M B A F D)$. Los mayores motivos para practicar AF fueron los relacionados con el gusto por la actividad y por las sensaciones positivas que les transmitía. La mayoría de los ítems sobre los motivos para la actividad física y el deporte (AFD), obtuvieron una puntuación alta. La falta de actividades físicas adaptadas y la falta de espacios próximos donde practicar AF, fueron las barreras más destacadas para la AFD. Cabe resaltar que los participantes no percibían excesivas barreras en los ítems para la práctica de la actividad deportiva $(<1,2 \pm 1,3)$. Salvo en ítems muy puntuales, no se obtuvieron diferencias significativas en función del sexo, el origen de la discapacidad y los años de experiencia en la práctica de otras modalidades deportivas previas a la práctica del goalball. Todo ello, puede deberse al hecho de que los participantes fueran personas con discapacidad visual asiduos practicantes de goalball, condicionando de este modo los resultados obtenidos.

\section{Palabras clave}

Deporte adaptado; salud; actividad física; hábitos.

\begin{abstract}
The objective of this study was to analyze the motives and barriers for practicing physical activity (PA) in visually impaired goalball players. 25 adults $(32.7 \pm 11.9$ years $)$ participated in this study, of which 14 were women $(31.3 \pm 12.0$ years $)$ and 11 were men $(34.4 \pm 12.2$ years). All participants had Federal license in force issued by the Spanish Federation of Sports for the Blind. Participants completed the Motives and Barriers to Physical Activity and Sport (MBAFD) questionnaire. The main reasons for practicing PA were related to the pleasure for the activity and the produced positive feelings. Most of the items related with the reasons for doing PA and sport obtained a high score. The lack of adapted physical activities and the lack of nearby spaces to practice PA were the most prominent barriers for doing PA and sport. It should be noted that the participants did not perceive excessive barriers in the items for the sports practice $(<1.2 \pm 1.3)$. Except in very specific items, no significant differences were obtained according to sex, the origin of the disability or years of experience in the practice of other sports modalities prior to the practice of goalball. This may be due to the fact that the participants in study were visually impaired goalball players, thus conditioning the results obtained.
\end{abstract}

\section{Key words}

Adapted sport; health; physical activity; habits.

Para citar este artículo utilice la siguiente referencia: Avalos Solitario, A.B.; Iturricastillo Urteaga, A.; Castillo Alvira, D.; Romaratezabala Aldasoro, E.; Rodríguez Negro, J.; Aritzeta de Pérdigo, I.; Yanci Irigoyen, J. (2021). Motivos y barreras para la práctica de actividad física percibidas por jugadores de goalball con discapacidad visual. Sportis Sci J, 7 (1), 43-66. https://doi.org/10.17979/sportis.2021.7.1.6713 
Artículo Original. Motivos y barreras para la práctica de actividad física percibidas por jugadores de goalball con discapacidad visual. Vol. 7 , n. $^{\circ}$; p. 43-66, enero 2021. A Coruña. España ISSN 2386-8333
\end{abstract}

\title{
Introducción
}

Se ha expuesto que una adecuada práctica de actividad física (AF) es beneficiosa para la población general ya que ayuda a mejorar la capacidad cardiovascular (Wilson, Ellison y Cable, 2016), la función neuromuscular y el sistema músculo-esquelético (Garber, Blissmer, Deschenes, Franklin, Lamonte, Lee, Nieman y Swain, 2011), entre otros aspectos. Se ha descrito que una práctica correctamente realizada y estructurada también presenta beneficios en el estado psicológico de los practicantes (Bonet, Parrado y Capdevila, 2017). En el ámbito de la discapacidad, los beneficios de la AF también se encuentran presentes, ya que en la literatura científica existente se pone de manifiesto que la AF tiene múltiples beneficios en la salud física (Brizuela, Romero y Beltrán, 2016; Smith, Kirby, Skinner, Wightman, Lucas y Foster, 2019; Heras, Reina, Roldán, Iturricastillo, Rodríguez-Negro y Yanci, 2019), en la salud mental (Montalvo y Jalomo, 2016) y en el plano social (Place y Hodge, 2001) de las personas con discapacidad. Concretamente en personas con discapacidad visual (DV), se ha observado que la AF reporta beneficios, por ejemplo, en la motricidad o en la capacidad cardiovascular (Çolak, Bamaç, Aydin, Meriç y Özbek, 2004). De la misma forma, se ha observado que las personas con DV practicantes asiduos de AF tienen menores porcentajes de grasa corporal frente a personas con DV sedentarias (Çıtak, Aki y Ergun, 2009), indicando de esta forma una mejor salud en aquellas personas con DV activas. Por lo tanto, la investigación actual apunta a que la AF es una herramienta esencial para la salud de las personas con discapacidad.

Sin embargo, a pesar de la evidencia científica existente sobre los beneficios derivados de la práctica de AF para personas con cualquier tipo de discapacidad, muchos estudios han constatado que los niveles de práctica de AF de las personas con discapacidad son netamente inferiores a los de personas sin discapacidad (Aviram, Harries, Rabani, Amro, Nammourah, Al-Jarrah, Raanan, Hutzler y Bar-Haim, 2019; McKeon, Slevin y Taggart, 2013; Dairo, Collett, Dawes y Oskrochi, 2016), e inferiores incluso a las recomendaciones realizadas por distintas instituciones (Petro, Herazo y Pinillos, 2019). Este menor nivel de práctica de AF de las personas con discapacidad ha sido constatado en distintas edades, sexos y tipos de

Para citar este artículo utilice la siguiente referencia: Avalos Solitario, A.B.; Iturricastillo Urteaga, A.; Castillo Alvira, D.; Romaratezabala Aldasoro, E.; Rodríguez Negro, J.; Aritzeta de Pérdigo, I.; Yanci Irigoyen, J. (2021). Motivos y barreras para la práctica de actividad física percibidas por jugadores de goalball con discapacidad visual. Sportis Sci J, 7 (1), 43-66. https://doi.org/10.17979/sportis.2021.7.1.6713 
Artículo Original. Motivos y barreras para la práctica de actividad física percibidas por jugadores de goalball con discapacidad visual. Vol. 7, n. $^{\circ}$; p. 43-66, enero 2021. A Coruña. España ISSN 2386-8333

discapacidad (Ramírez, Sanz y Figueroa de la Paz, 2007; Wouters, Evenhuis y Hilgenkamp, 2018; Smith, Jackson, Pardhan, López-Sánchez, Hu, Cao, Vancampfort, Koyanagi, Stubbs, Firth y Yang, 2019; Sadowska y Krzepota, 2015). Por ejemplo, en un estudio reciente (Wouters et al., 2018) se observó que los niños de 2 a 18 años con discapacidad intelectual (DI) no alcanzaban los 60 min al día de AF recomendada por la Organización Mundial de la Salud (OMS, 2019). En la misma línea, Aviram et al. (2019) evaluaron el nivel de AF en personas con parálisis cerebral (PC) y personas sin PC, constatando que las personas con PC tenían una menor práctica de AF y unos mayores valores de sedentarismo. Ramirez et al. (2007), en un estudio con población adulta con discapacidad, observaron que únicamente el $29 \%$ de los participantes realizaba más de los 60 min diarios recomendados por la OMS y que el $51 \%$ de las mujeres y el $40,7 \%$ de los hombres eran sedentarios. Por otro lado, en un estudio realizado con mujeres adultas con discapacidad (Gallego, Aguilar-Parra, Cangas, Pérez-Escobar y Barrera, 2014), reportaron que el 44,6\% de las participantes no realizaba ningún tipo de AF. Estos bajos niveles de práctica de AF también se han expuesto en personas con DV. Un estudio reciente (Smith et al., 2019) describió que los adultos con DV de más de 50 años eran más sedentarios que los adultos sin discapacidad, particularmente en el grupo de mujeres. Del mismo modo, Sadowska et al. (2015) observaron que el 68\% de las personas con DV encuestadas no llegaban a los niveles de práctica de AF recomendados por la OMS. El conocimiento científico existente parece evidenciar que las personas con discapacidad, y también las personas con DV, tienen menores niveles de práctica de AF y que son poblaciones con altos niveles de sedentarismo.

Con el fin de poder actuar de forma eficiente en el aumento de los niveles de práctica de AF de las personas con discapacidad, resulta indispensable conocer qué aspectos motivan y qué dificultades perciben las personas con discapacidad para la práctica de actividad física y deportiva (Ramírez, Sanz y Figueroa, 2007; Jaarsma, Geertzen, De Jong, Dijkstra y Dekker, 2014; Muñoz, Garrote, Rojas y Sánchez, 2017). En este sentido, diferentes estudios han analizado las barreras y los motivos percibidos por personas con diferentes discapacidades (Ramírez, Sanz y Figueroa, 2007; Lazcano, Ortuza y Romero, 2017; Torralba, Braz y Rubio, 2017; Abellán y Januário, 2017; Mendia, Iturricastillo, Rodríguez-Negro, Romarate y Yanci,

Para citar este artículo utilice la siguiente referencia: Avalos Solitario, A.B.; Iturricastillo Urteaga, A.; Castillo Alvira, D.; Romaratezabala Aldasoro, E.; Rodríguez Negro, J.; Aritzeta de Pérdigo, I.; Yanci Irigoyen, J. (2021). Motivos y barreras para la práctica de actividad física percibidas por jugadores de goalball con discapacidad visual. Sportis Sci J, 7 (1), 43-66. https://doi.org/10.17979/sportis.2021.7.1.6713 
Artículo Original. Motivos y barreras para la práctica de actividad física percibidas por jugadores de goalball con discapacidad visual. Vol. 7 , n. ${ }^{\circ}$; p. 43-66, enero 2021. A Coruña. España ISSN 2386-8333

2020), como personas con discapacidad física (DF) (Muñoz, Garrote y Sánchez, 2017; Torralba et al., 2017), DI y PC (Verschuren, Wiart, Hermans y Ketelaar; 2012, Conchar, Bantjes, Swartz y Derman, 2016; Mendia, Iturricastillo, Rodríguez-Negro, Romarate y Yanci, 2020; Muñoz, Garrote y Sánchez, 2017) para la práctica de la AF. Concretamente, en personas con DV también existen estudios que analizan los motivos y las barreras para la práctica de AF (Jaarsma, Dekker, Koopmans, Dijkstra y Geertzen, 2014; Phoenix, Giffin y Smith, 2015). Jaarsma et al. (2014) expusieron que las barreras percibidas por personas con DV mayores de 18 años fueron el transporte y la falta de actividades ofertadas en un entorno cercano. Por su parte, Phoenix et al. (2015) observaron nuevamente que el transporte y la des-información de las AF constituían las barreras fundamentales. A pesar de que existen estudios sobre los motivos y las barreras para la práctica de $\mathrm{AF}$ en personas con $\mathrm{DV}$, normalmente estos trabajos se han realizado en población sedentaria. Puede ser necesario conocer cuáles son los motivos y las barreras para la práctica de AF en personas deportistas con DV, ya que el contexto, el entorno social, cultural o el nivel de práctica deportiva habitual pueden condicionar los motivos y las barreras percibidas (Mendia et al., 2020).

Por lo tanto, el objetivo de este estudio fue, por un lado, analizar los motivos y las barreras para la práctica de AF percibidas por deportistas de goalball con DV, y por otro lado, comparar las diferencias en función del sexo, del origen de la discapacidad y de los años de experiencia practicando alguna modalidad deportiva previa a la práctica del goalball.

\section{Método}

\section{Participantes}

En este estudio participaron 25 deportistas adultos con DV $(32,7 \pm 11,9$ años), de los cuales 14 eran mujeres $(31,3 \pm 12,0$ años) y 11 eran hombres (34,4 $\pm 12,2$ años). Se realizó una selección de la muestra por conveniencia. En la Tabla 1 se presenta la distribución de participantes atendiendo al sexo, al origen de la discapacidad y a los años de experiencia en la práctica deportiva. Todos los participantes en el estudio tenían licencia federativa en vigor expedida por la Federación Española de Deportes para Ciegos, concretamente en la modalidad de goalball. Los criterios de inclusión considerados en este estudio fueron ser

Para citar este artículo utilice la siguiente referencia: Avalos Solitario, A.B.; Iturricastillo Urteaga, A.; Castillo Alvira, D.; Romaratezabala Aldasoro, E.; Rodríguez Negro, J.; Aritzeta de Pérdigo, I.; Yanci Irigoyen, J. (2021). Motivos y barreras para la práctica de actividad física percibidas por jugadores de goalball con discapacidad visual. Sportis Sci J, 7 (1), 43-66. https://doi.org/10.17979/sportis.2021.7.1.6713 
Artículo Original. Motivos y barreras para la práctica de actividad física percibidas por jugadores de goalball con discapacidad visual. Vol. 7 , n. $^{\circ}$; p. 43-66, enero 2021. A Coruña. España ISSN 2386-8333

mayores de edad y disponer de licencia federativa oficial en vigor para poder participar en la Liga Nacional de Goalball. Todos los participantes fueron informados de los procedimientos y la metodología del estudio antes de cumplimentar el cuestionario y participaron libremente en el estudio. El estudio siguió las pautas marcadas en la Declaración de Helsinki (2013) y fue aprobado por el Comité de Ética para la Investigación con Seres Humanos (CEISH, código M10_2019_058) de la Universidad del País Vasco (UPV/EHU).

Tabla 1. Participantes del estudio $(n=25)$ atendiendo al sexo, al origen de la discapacidad y a los años de experiencia en la práctica deportiva.

\begin{tabular}{|c|c|c|c|c|c|c|c|}
\hline & \multicolumn{3}{|c|}{ Origen de la discapacidad } & \multicolumn{4}{|c|}{ Años de experiencia en la práctica deportiva } \\
\hline & Congénita & Adquirida & $\mathrm{NS} / \mathrm{NC}$ & 0-3 años exp. & $\begin{array}{c}\text { 4-8 años } \\
\text { exp. }\end{array}$ & $\begin{array}{l}>9 \text { años } \\
\text { exp. }\end{array}$ & Total \\
\hline Mujeres & 10 & 3 & 1 & 2 & 8 & 4 & 14 \\
\hline Hombres & 7 & 4 & 0 & 1 & 3 & 7 & 11 \\
\hline Total & 17 & 7 & 1 & 3 & 11 & 11 & 25 \\
\hline
\end{tabular}

$\mathrm{NS} / \mathrm{NC}=$ no sabe $/$ no contesta, años exp. $=$ años de experiencia.

\section{Procedimientos}

Durante los meses de noviembre y diciembre de 2019 y enero de 2020, en el transcurso de diferentes entrenamientos y competiciones de goalball, se pasó la encuesta Motivos y Barreras para la práctica de Actividad Física y Deporte (MBAFD) a todos los participantes en el estudio, con el fin de conocer la percepción sobre los motivos y las barreras percibidas para la práctica del goalball. La encuesta fue cumplimentada por cada participante de manera escrita e individual y en presencia de uno de los investigadores. A los deportistas que no tenían posibilidad de poder leer el cuestionario, el investigador leyó cada uno de los ítems y registró la respuesta dada por el deportista. En caso de que los participantes lo solicitaran, los investigadores realizaron las aclaraciones oportunas a las cuestiones planteadas en la encuesta.

Para citar este artículo utilice la siguiente referencia: Avalos Solitario, A.B.; Iturricastillo Urteaga, A.; Castillo Alvira, D.; Romaratezabala Aldasoro, E.; Rodríguez Negro, J.; Aritzeta de Pérdigo, I.; Yanci Irigoyen, J. (2021). Motivos y barreras para la práctica de actividad física percibidas por jugadores de goalball con discapacidad visual. Sportis Sci J, 7 (1), 43-66. https://doi.org/10.17979/sportis.2021.7.1.6713 


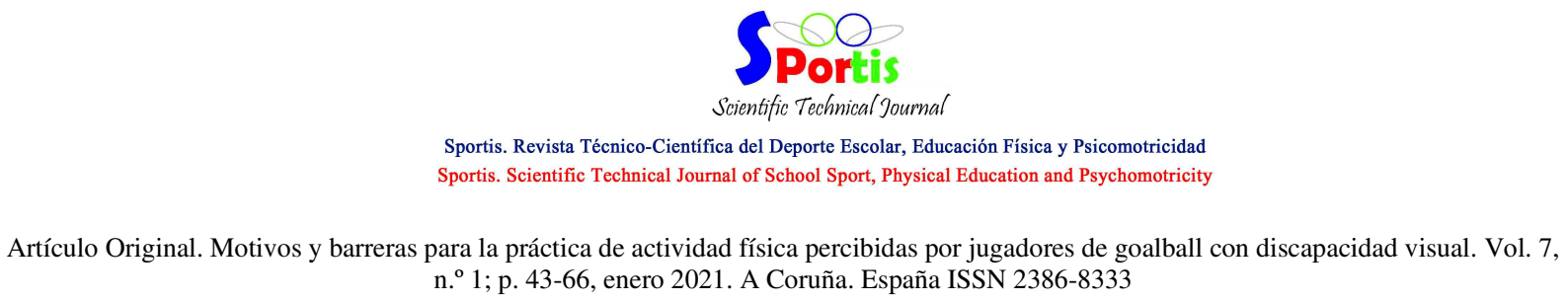

\section{Mediciones}

Cuestionario Motivos y Barreras para la práctica de Actividad Física y Deporte $(M B A F D)$ : Para identificar los motivos y las barreras para la práctica del goalball, se empleó el cuestionario MBAFD, validado anteriormente por Koustik (2016) para población adulta y utilizado recientemente por Mendia et al. (2019) en un estudio de características similares al presente trabajo. La encuesta utilizada estaba dividida en 2 bloques con un total de 60 ítems o preguntas. El primer bloque hacía referencia a los motivos para la práctica del goalball y estaba compuesto por un total de 26 ítems o preguntas (ítems motivos 1-26). El segundo bloque hacía referencia a las barreras para la práctica del goalball y estaba compuesto por un total de 28 ítems o preguntas (ítems-barreras 1-28). El ítem o pregunta número 28, "No existe una amplia oferta de actividades físicas adaptadas", que no estaba contemplado en el cuestionario original, se incluyó con el fin de conocer específicamente si la oferta de actividades físicas adaptadas disponibles era una barrera. Las respuestas a los ítems eran en una escala de tipo Likert ( 0 = nada y $3=$ mucho $)$, que incluía también una posible opción de no sabe/no contesta (NS/NC), no incluida para el análisis estadístico.

\section{Análisis estadístico}

Los resultados se presentan como media \pm desviación típica (DT). Para el cálculo de las diferencias de medias en función del sexo (hombres y mujeres) o entre los grupos atendiendo al origen de la discapacidad (congénita o adquirida), se utilizó el estadístico U de Mann Whitney. Con el fin de analizar las diferencias de medias entre los grupos atendiendo a los años de experiencia en la práctica deportiva (0-3 años, 4-8 años y >9 años) se utilizó el estadístico Kruskal Wallis. El análisis estadístico se realizó con el programa Statistical Package for Social Sciences (SPSS Inc, versión 23,0 Chicago, IL, EE.UU.). La significatividad estadística fue establecida en $p<0,05$.

\section{Resultados}

En las Tablas 2 y 3 se presentan los resultados de los motivos y de las barreras percibidas por los participantes a la hora de practicar AF, tanto para el total de la muestra

Para citar este artículo utilice la siguiente referencia: Avalos Solitario, A.B.; Iturricastillo Urteaga, A.; Castillo Alvira, D.; Romaratezabala Aldasoro, E.; Rodríguez Negro, J.; Aritzeta de Pérdigo, I.; Yanci Irigoyen, J. (2021). Motivos y barreras para la práctica de actividad física percibidas por jugadores de goalball con discapacidad visual. Sportis Sci J, 7 (1), 43-66. https://doi.org/10.17979/sportis.2021.7.1.6713 
Artículo Original. Motivos y barreras para la práctica de actividad física percibidas por jugadores de goalball con discapacidad visual. Vol. 7 , n. ${ }^{\circ}$; p. 43-66, enero 2021. A Coruña. España ISSN 2386-8333

como para el grupo de mujeres y de hombres. Los resultados obtenidos en los motivos para la práctica muestran que, para el total de la muestra, los ítems "Por diversión" (M7), "Porque disfruto aprendiendo cosas nuevas" (M8), "Porque me gusta hacer esta actividad física" (M11) y "Por las sensaciones positivas que me produce realizar AF" (M19) presentaron los valores más altos. Por el contrario, los ítems "Para recuperarme de una enfermedad o lesión" (M1), "Para pasar más tiempo con mi pareja" (M15), "Por recomendación médica" (M22) y "Para compartir tiempo con mis hijo@s" (M26), presentaron los valores más bajos. Con respecto a las barreras percibidas, los ítems que presentaron los valores más altos por los participantes fueron "No hay espacios próximos donde practicar" (M10) y "No existe una amplia oferta de actividades físicas adaptadas" (B28). El resto de ítems con respecto a las barreras percibidas tuvieron una media menor a 1. En referencia a la comparativa entre mujeres y hombres, se obtuvieron diferencias significativas $(p<0,05)$ únicamente en el ítem "No soy capaz de generar tiempo para mî” (M12).

Tabla 2. Resultados de los motivos percibidos para la práctica de actividad física (AF) por el total de participantes, por el grupo de mujeres y por el grupo de hombres.

\begin{tabular}{lccc}
\hline & Todos & Mujeres & Hombres \\
\hline Motivos & & & \\
M1 Para recuperarme de una enfermedad o lesión & $0,2 \pm 0,5$ & $0,1 \pm 0,3$ & $0,3 \pm 0,6$ \\
M2 Para controlar el peso & $0,5 \pm 0,9$ & $0,3 \pm 0,6$ & $0,8 \pm 1,2$ \\
M3 Para estar en forma & $1,9 \pm 1,0$ & $1,6 \pm 1,1$ & $2,4 \pm 0,8$ \\
M4 Porque haciendo AF me siento bien conmigo & $2,6 \pm 0,7$ & $2,7 \pm 0,6$ & $2,5 \pm 0,7$ \\
mism @ & $1,6 \pm 1,2$ & $1,8 \pm 1,3$ & $1,4 \pm 1,2$ \\
M5 Porque es un tiempo solo para mi & $2,2 \pm 0,9$ & $2,0 \pm 0,9$ & $2,4 \pm 0,9$ \\
M6 Para encontrarme con mis amistades & $2,8 \pm 0,4$ & $2,9 \pm 0,4$ & $2,7 \pm 0,5$ \\
M7 Por diversión & $2,8 \pm 0,5$ & $2,7 \pm 0,6$ & $2,8 \pm 0,4$ \\
M8 Porque disfruto aprendiendo cosas nuevas & $2,2 \pm 0,8$ & $2,1 \pm 0,8$ & $2,4 \pm 0,8$ \\
M9 Para mejorar mi salud física & $2,5 \pm 0,6$ & $2,6 \pm 0,6$ & $2,3 \pm 0,6$ \\
M10 Para mejorar mi bienestar mental & $2,9 \pm 0,4$ & $2,9 \pm 0,5$ & $2,9 \pm 0,3$ \\
M11 Porque me gusta hacer esta AF & $2,4 \pm 0,9$ & $2,8 \pm 0,6$ & $1,8 \pm$
\end{tabular}

Para citar este artículo utilice la siguiente referencia: Avalos Solitario, A.B.; Iturricastillo Urteaga, A.; Castillo Alvira, D.; Romaratezabala Aldasoro, E.; Rodríguez Negro, J.; Aritzeta de Pérdigo, I.; Yanci Irigoyen, J. (2021). Motivos y barreras para la práctica de actividad física percibidas por jugadores de goalball con discapacidad visual. Sportis Sci J, 7 (1), 43-66. https://doi.org/10.17979/sportis.2021.7.1.6713 
Artículo Original. Motivos y barreras para la práctica de actividad física percibidas por jugadores de goalball con discapacidad visual. Vol. 7 , n. ${ }^{\circ} 1$; p. 43-66, enero 2021. A Coruña. España ISSN 2386-8333

M13 Para mejorar mi estado de ánimo

M14 Para relacionarme con otras personas

M15 Para pasar más tiempo con mi pareja

M16 Porque me siento mal si no practico ninguna $\mathrm{AF}$

M17 Porque me gusta superar retos

M18 Porque quiero que forme parte de mi estilo de vida

M19 Por las sensaciones positivas que me produce realizar AF

M20 Porque me permite pasar tiempo fuera de casa

M21 Por recomendación de mi entorno

M22 Por recomendación medica

M23 Para mejorar mi aspecto físico

M24 Para mejorar mi capacidad física

M25 Para desconectar un rato

M26 Para compartir actividades con mis hij@s

$$
2,2 \pm 0,7
$$$$
2,3 \pm 0,7
$$

$2,2 \pm 0,6$

$2,5 \pm 0,6$

$2,6 \pm 0,6$

$2,4 \pm 0,7$

$0,3 \pm 0,7$

$0,4 \pm 0,9$

$0,3 \pm 0,5$

$2,0 \pm 1,1$

$2,0 \pm 1,2$

$1,9 \pm 0,8$

$2,5 \pm 0,7$

$2,9 \pm 0,5$

$2,1 \pm 0,7$

$2,5 \pm 0,7$

$2,6 \pm 0,7$

$2,3 \pm 0,6$

\section{$2,9 \pm 0,3 \quad 2,9 \pm 0,4 \quad 2,9 \pm 0,3$}

$1,5 \pm 1,2 \quad 1,7 \pm 1,1 \quad 1,2 \pm 1,2$

$1,3 \pm 1,3 \quad 1,1 \pm 1,2 \quad 1,4 \pm 1,4$

$0,3 \pm 0,7 \quad 0,4 \pm 0,9 \quad 0,2 \pm 0,4$

$1,0 \pm 1,1 \quad 0,9 \pm 1,1 \quad 1,3 \pm 1,0$

$2,1 \pm 0,8 \quad 2,2 \pm 0,9 \quad 2,0 \pm 0,8$

$2,6 \pm 0,6 \quad 2,6 \pm 0,6 \quad 2,5 \pm 0,5$

$0,0 \pm 0,2 \quad 0,0 \pm 0,0 \quad 0,1 \pm 0,3$

$* \mathrm{p}<0.05$ diferencias significativas con respecto al grupo de mujeres.

Tabla 3. Resultados de las barreras percibidas para la práctica de actividad física (AF) por el total de participantes, por el grupo de mujeres y por el grupo de hombres.

\begin{tabular}{lccc}
\hline & Todos & Mujeres & Hombres \\
\hline Barreras & & & \\
B1 Temor a hacerme daño & $0,5 \pm 0,9$ & $0,6 \pm 1,1$ & $0,4 \pm 0,7$ \\
B2 Me da vergüenza que me vean haciendo ejercicio & $0,1 \pm 0,3$ & $0,1 \pm 0,4$ & $0,0 \pm 0,0$ \\
B3 No me siento a gusto con mi cuerpo & $0,2 \pm 0,6$ & $0,2 \pm 0,4$ & $0,3 \pm 0,6$ \\
B4 Mi empleo actual me lo impide & $0,4 \pm 0,8$ & $0,6 \pm 0,9$ & $0,2 \pm 0,4$ \\
B5 Soy demasiado mayor & $0,0 \pm 0,2$ & $0,0 \pm 0,0$ & $0,1 \pm 0,3$ \\
B6 Estoy cansad@ & $0,2 \pm 0,5$ & $0,3 \pm 0,6$ & $0,2 \pm 0,4$ \\
B7 Es demasiado caro & $0,0 \pm 0,2$ & $0,0 \pm 0,0$ & $0,1 \pm 0,3$ \\
B8 Las personas que dirigen la actividad no son & $0,6 \pm 1,1$ & $0,6 \pm 1,3$ & $0,4 \pm 0,8$
\end{tabular}

Para citar este artículo utilice la siguiente referencia: Avalos Solitario, A.B.; Iturricastillo Urteaga, A.; Castillo Alvira, D.; Romaratezabala Aldasoro, E.; Rodríguez Negro, J.; Aritzeta de Pérdigo, I.; Yanci Irigoyen, J. (2021). Motivos y barreras para la práctica de actividad física percibidas por jugadores de goalball con discapacidad visual. Sportis Sci J, 7 (1), 43-66. https://doi.org/10.17979/sportis.2021.7.1.6713 
Artículo Original. Motivos y barreras para la práctica de actividad física percibidas por jugadores de goalball con discapacidad visual. Vol. 7 , n. ${ }^{\circ} 1$; p. 43-66, enero 2021. A Coruña. España ISSN 2386-8333

adecuados

B9 Los espacios donde puedo practicar no son adecuados

B10 No hay espacios próximos donde practicar

B11 No me gusta

B12 No soy capaz de generar tiempo para mi

B13 Me da pereza

B14 No sé dónde hacer ejercicio

B15 Tengo que cuidar a mis hij@s

B16 No me siento capaz

B17 No estoy en forma

B18 Dificultades de transporte

B19 No tengo con quien hacer ejercicio

B20 Tengo que ocuparme de las tareas del hogar

B21 Tengo mal estado de salud

B22 Horarios incompatibles

B23 No tengo costumbre

B24 No se oferta la actividad que me gusta

B25 No veo necesario hacer ejercicio

B26 Tengo que cuidar a familiares mayores

B27 Considero mi discapacidad una barrera

B28 No existe una amplia oferta de AFs adaptadas
$0,6 \pm 1,0 \quad 0,8 \pm 1,1 \quad 0,4 \pm 0,8$

$1,2 \pm 1,3 \quad 1,3 \pm 1,1 \quad 1,1 \pm 1,4$

$0,0 \pm 0,2 \quad 0,0 \pm 0,0 \quad 0,1 \pm 0,3$

$0,4 \pm 0,5 \quad 0,4 \pm 0,5 \quad 0,4 \pm 0,5$

$0,2 \pm 0,5 \quad 0,1 \pm 0,3 \quad 0,5 \pm 0,7$

$0,4 \pm 0,8 \quad 0,4 \pm 0,9 \quad 0,3 \pm 0,6$

$0,1 \pm 0,4 \quad 0,0 \pm 0,0 \quad 0,2 \pm 0,6$

$0,0 \pm 0,0 \quad 0,0 \pm 0,0 \quad 0,0 \pm 0,0$

$0,4 \pm 0,5 \quad 0,4 \pm 0,5 \quad 0,4 \pm 0,5$

$0,9 \pm 1,1 \quad 0,9 \pm 1,2 \quad 0,4 \pm 1,0$

$0,2 \pm 0,5 \quad 0,2 \pm 0,4 \quad 0,3 \pm 0,6$

$0,5 \pm 0,8 \quad 0,4 \pm 0,6 \quad 0,6 \pm 1,0$

$0,3 \pm 0,7 \quad 0,4 \pm 0,9 \quad 0,1 \pm 0,3$

$0,7 \pm 0,8 \quad 0,9 \pm 1,0 \quad 0,4 \pm 0,5$

$0,2 \pm 0,4 \quad 0,2 \pm 0,4 \quad 0,1 \pm 0,3$

$0,1 \pm 0,3 \quad 0,0 \pm 0,0 \quad 0,2 \pm 0,4$

$0,0 \pm 0,0 \quad 0,0 \pm 0,0 \quad 0,0 \pm 0,0$

$0,0 \pm 0,2 \quad 0,0 \pm 0,0 \quad 0,1 \pm 0,3$

$0,4 \pm 0,8 \quad 0,6 \pm 1,0 \quad 0,1 \pm 0,3$

$1,2 \pm 1,2 \quad 1,2 \pm 1,3 \quad 1,1 \pm 1,0$

$* \mathrm{p}<0.05$ diferencias significativas con respecto al grupo de mujeres.

En las Tablas 4 y 5 se presentan los resultados de los motivos y de las barreras para la práctica de $\mathrm{AF}$ percibidas por los jugadores de goalball atendiendo al origen de la discapacidad (congénita o adquirida) y a los años de experiencia en la práctica deportiva. En el caso del origen de la discapacidad, únicamente se observaron diferencias significativas ( $\mathrm{p}<$ 0,05) entre el grupo de discapacidad congénita y el de adquirida en el ítem "Me da vergüenza que me vean haciendo ejercicio" (B2). Con respecto a los años de experiencia practicando alguna modalidad deportiva, únicamente se observaron diferencias significativas $(\mathrm{p}<0,05)$

Para citar este artículo utilice la siguiente referencia: Avalos Solitario, A.B.; Iturricastillo Urteaga, A.; Castillo Alvira, D.; Romaratezabala Aldasoro, E.; Rodríguez Negro, J.; Aritzeta de Pérdigo, I.; Yanci Irigoyen, J. (2021). Motivos y barreras para la práctica de actividad física percibidas por jugadores de goalball con discapacidad visual. Sportis Sci J, 7 (1), 43-66. https://doi.org/10.17979/sportis.2021.7.1.6713 
Artículo Original. Motivos y barreras para la práctica de actividad física percibidas por jugadores de goalball con discapacidad visual. Vol. 7 , n. $^{\circ}$; p. 43-66, enero 2021. A Coruña. España ISSN 2386-8333

entre el grupo de "4-8 años de experiencia" y el grupo de ">9 años de experiencia” en el ítem "Porque me gusta superar retos" (M17).

Tabla 4. Resultados de los motivos percibidos para la práctica de actividad física (AF) según el origen de la discapacidad (congénita y adquirida) y según los años de experiencia practicando otras disciplinas deportivas.

D.

Congénita Adquirida experiencia experiencia experiencia

\section{Motivos}

M1 Para recuperarme de una enfermedad o lesión

$\begin{array}{lllll}0,1 \pm 0,3 & 0,2 \pm 0,7 & 0,1 \pm 0,3 & 0,3 \pm 0,6 & 0,0 \pm 0,0 \\ 0,7 \pm 1,0 & 0,2 \pm 0,7 & 0,5 \pm 1,0 & 0,7 \pm 0,9 & 0,0 \pm 0,0 \\ 1,9 \pm 1,0 & 1,7 \pm 1,2 & 2,2 \pm 1,0 & 1,7 \pm 1,1 & 1,7 \pm 1,2\end{array}$

M3 Para estar en forma

M4 Porque haciendo AF me siento bien conmigo mismo/a

M5 Porque es un tiempo solo para mi

M6 Para encontrarme con mis amistades

M7 Por diversión

M8 Porque disfruto aprendiendo cosas nuevas

M9 Para mejorar mi salud física

M10 Para mejorar mi bienestar mental

M11 Porque me gusta hacer esta actividad física

M12 Para eliminar el estrés

M13 Para mejorar mi estado de ánimo

M14 Para relacionarme con otras personas

M15 Para pasar más tiempo con mi pareja

$\begin{array}{lllll}1,6 \pm 1,2 & 1,5 \pm 1,4 & 1,5 \pm 1,3 & 2,1 \pm 1,1 & 0,3 \pm 0,6 \\ 2,4 \pm 0,8 & 1,7 \pm 1,0 & 2,3 \pm 0,8 & 2,2 \pm 0,9 & 1,7 \pm 1,5 \\ 2,8 \pm 0,4 & 2,7 \pm 0,5 & 2,8 \pm 0,4 & 2,8 \pm 0,4 & 2,7 \pm 0,6 \\ 2,9 \pm 0,3 & 2,5 \pm 0,8 & 2,9 \pm 0,3 & 2,6 \pm 0,7 & 2,7 \pm 0,6 \\ 2,2 \pm 0,8 & 2,1 \pm 1,0 & 2,5 \pm 0,8 & 2,1 \pm 0,8 & 2,0 \pm 1,0 \\ 2,6 \pm 0,6 & 2,2 \pm 0,7 & 2,5 \pm 0,5 & 2,5 \pm 0,8 & 2,3 \pm 0,6 \\ 2,8 \pm 0,5 & 3,0 \pm 0,0 & 2,9 \pm 0,3 & 2,8 \pm 0,6 & 3,0 \pm 0,0 \\ 2,4 \pm 0,9 & 2,1 \pm 1,0 & 2,0 \pm 1,0 & 2,6 \pm 0,7 & 1,7 \pm 1,2 \\ 2,2 \pm 0,6 & 2,2 \pm 0,7 & 2,5 \pm 0,5 & 2,2 \pm 0,8 & 1,7 \pm 0,6 \\ 2,4 \pm 0,7 & 2,6 \pm 0,5 & 2,5 \pm 0,8 & 2,5 \pm 0,5 & 2,7 \pm 0,6 \\ 0,4 \pm 0,9 & 0,1 \pm 0,3 & 0,2 \pm 0,4 & 0,5 \pm 1,0 & 0,0 \pm 0,0\end{array}$

Para citar este artículo utilice la siguiente referencia: Avalos Solitario, A.B.; Iturricastillo Urteaga, A.; Castillo Alvira, D.; Romaratezabala Aldasoro, E.; Rodríguez Negro, J.; Aritzeta de Pérdigo, I.; Yanci Irigoyen, J. (2021). Motivos y barreras para la práctica de actividad física percibidas por jugadores de goalball con discapacidad visual. Sportis Sci J, 7 (1), 43-66. https://doi.org/10.17979/sportis.2021.7.1.6713 
Artículo Original. Motivos y barreras para la práctica de actividad física percibidas por jugadores de goalball con discapacidad visual. Vol. 7, n. $^{\circ} 1$; p. 43-66, enero 2021. A Coruña. España ISSN 2386-8333

M16 Porque me siento mal si no practico ninguna $\mathrm{AF}$

$$
1,9 \pm 1,0 \quad 2,0 \pm 1,2 \quad 2,3 \pm 0,8 \quad 1,6 \pm 1,1 \quad 2,0 \pm 1,7
$$

M17 Porque me gusta superar retos

$$
2,5 \pm 0,7 \quad 2,5 \pm 0,7 \quad 2,5 \pm 0,7 \# \quad 2,8 \pm 0,4 \quad 1,7 \pm 1,2
$$

M18 Porque quiero que forme parte de mi estilo de vida

$$
2,4 \pm 0,6 \quad 2,4 \pm 0,7 \quad 2,5 \pm 0,8 \quad 2,6 \pm 0,5 \quad 1,7 \pm 0,6
$$

M19 Por las sensaciones positivas que me produce realizar AF

$2,8 \pm 0,4 \quad 3,0 \pm 0,0 \quad 3,0 \pm 0,0 \quad 2,7 \pm 0,5 \quad 3,0 \pm 0,0$

M20 Porque me permite pasar tiempo fuera de casa

$1,4 \pm 1,1 \quad 1,5 \pm 1,3 \quad 1,8 \pm 1,2 \quad 1,5 \pm 1,1 \quad 0,3 \pm 0,6$

M21 Por recomendación de mi entorno

$\begin{array}{lllll}1,2 \pm 1,3 & 1,1 \pm 1,4 & 1,7 \pm 1,4 & 1,0 \pm 1,2 & 0,7 \pm 1,2 \\ 0,2 \pm 0,6 & 0,0 \pm 0,0 & 0,4 \pm 0,9 & 0,1 \pm 0,3 & 0,7 \pm 1,2\end{array}$

M22 Por recomendación medica

$$
0,9 \pm 1,1 \quad 1,2 \pm 1,0 \quad 1,3 \pm 1,2 \quad 0,9 \pm 1,0 \quad 0,7 \pm 0,6
$$
aspecto físico

M24 Para mejorar mi capacidad física

$2,1 \pm 0,8 \quad 2,0 \pm 0,9 \quad 2,0 \pm 0,8 \quad 2,2 \pm 0,9 \quad 2,3 \pm 1,2$

M25 Para desconectar un rato

$$
2,7 \pm 0,5 \quad 2,4 \pm 0,7 \quad 2,7 \pm 0,5 \quad 2,6 \pm 0,5 \quad 2,0 \pm 1,0
$$

M26 Para compartir actividades con mis hijos/as
$0,1 \pm 0,2$
$0,0 \pm 0,0$
$0,0 \pm 0,0$
$0,1 \pm 0,3$
$0,0 \pm 0,0$

$\mathrm{D}=$ Discapacidad. $* p<0.05$ diferencias significativas con respecto grupo de discapacidad adquirida. \# $p<0.05$ diferencias significativas con respecto al grupo "4-8 años de experiencia" y ">9 años de experiencia”. 
Artículo Original. Motivos y barreras para la práctica de actividad física percibidas por jugadores de goalball con discapacidad visual. Vol. 7 , n. $^{\circ} 1$; p. 43-66, enero 2021. A Coruña. España ISSN 2386-8333

Tabla 5. Resultados de las barreras percibidas para la práctica de actividad física (AF) según el origen de la discapacidad (congénita y adquirida) y según los años de experiencia practicando otras disciplinas deportivas.

D.

Congénita Adquirida experiencia experiencia experiencia

\section{Barreras}

B1 Temor a hacerme daño

$$
0,5 \pm 0,9
$$

$0,2 \pm 0,5$

$0,7 \pm 1,0$

$0,2 \pm 0,4$

$1,0 \pm 1,7$

B2 Me da vergüenza que me vean haciendo ejercicio
$0,0 \pm 0,0$
$0,2 \pm 0,5^{*}$
$0,0 \pm 0,0$
$0,1 \pm 0,3$
$0,3 \pm 0,6$

B3 No me siento a gusto con mi cuerpo
$0,4 \pm 0,6$
$0,0 \pm 0,0$
$0,3 \pm 0,6$
$0,3 \pm 0,5$
$0,0 \pm 0,0$

B4 Mi empleo actual me lo impide

$\begin{array}{lllll}0,4 \pm 0,6 & 0,5 \pm 1,1 & 0,5 \pm 0,9 & 0,5 \pm 0,7 & 0,3 \pm 0,6 \\ 0,1 \pm 0,2 & 0,0 \pm 0,0 & 0,1 \pm 0,3 & 0,0 \pm 0,0 & 0,0 \pm 0,0 \\ 0,2 \pm 0,6 & 0,2 \pm 0,5 & 0,1 \pm 0,3 & 0,5 \pm 0,7 & 0,0 \pm 0,0 \\ 0,1 \pm 0,2 & 0,0 \pm 0,0 & 0,0 \pm 0,0 & 0,1 \pm 0,3 & 0,0 \pm 0,0\end{array}$

B5 Soy demasiado mayor

B6 Estoy cansado/a

$0,1 \pm 0,2$

$0,0 \pm 0,0$

$0,7 \pm 1,2 \quad 0,4 \pm 1,1$

$0,3 \pm 0,6$

$1,0 \pm 1,5$

$0,0 \pm 0,0$ adecuadas

B9 Los espacios donde puedo practicar no son adecuados

B10 No hay espacios próximos donde practicar
$0,7 \pm 1,0$
$0,5 \pm 1,1$
$0,8 \pm 1,1$
$0,6 \pm 1,0$
$0,0 \pm 0,0$

B11 No me gusta

$1,2 \pm 1,3$

$$
1,0 \pm 1,1
$$

$1,6 \pm 1,5$

$1,0 \pm 1,0$

$0,3 \pm 0,6$

B12 No soy capaz de generar tiempo para mi
$0,1 \pm 0,2$
$0,0 \pm 0,0$
$0,1 \pm 0,3$
$0,0 \pm 0,0$
$0,0 \pm 0,0$

$0,4 \pm 0,5$

$0,5 \pm 0,5$

$0,4 \pm 0,5$

$0,5 \pm 0,5$

$0,0 \pm 0,0$

B13 Me da pereza

B14 No sé dónde hacer ejercicio
$0,2 \pm 0,6$
$0,1 \pm 0,3$
$0,3 \pm 0,6$
$0,2 \pm 0,4$
$0,0 \pm 0,0$
$0,4 \pm 0,7 \quad 0,4 \pm 1,1$
$0,5 \pm 1,0$
$0,3 \pm 0,6$
$0,0 \pm 0,0$
$0,1 \pm 0,5 \quad 0,0 \pm 0,0$
$0,2 \pm 0,6$
$0,0 \pm 0,0$
$0,0 \pm 0,0$
$0,0 \pm 0,0$
$0,0 \pm 0,0$
$0,0 \pm 0,0$
$0,0 \pm 0,0$
$0,0 \pm 0,0$
$0,4 \pm 0,5$
$0,5 \pm 0,5$
$0,4 \pm 0,5$
$0,5 \pm 0,5$
$0,3 \pm 0,6$

B15 Tengo que cuidar a mis hijos/as

B16 No me siento capaz

B17 No estoy en forma

Para citar este artículo utilice la siguiente referencia: Avalos Solitario, A.B.; Iturricastillo Urteaga, A.; Castillo Alvira, D.; Romaratezabala Aldasoro, E.; Rodríguez Negro, J.; Aritzeta de Pérdigo, I.; Yanci Irigoyen, J. (2021). Motivos y barreras para la práctica de actividad física percibidas por jugadores de goalball con discapacidad visual. Sportis Sci J, 7 (1), 43-66. https://doi.org/10.17979/sportis.2021.7.1.6713 
Artículo Original. Motivos y barreras para la práctica de actividad física percibidas por jugadores de goalball con discapacidad visual. Vol. 7 , n. 1 ; p. 43-66, enero 2021. A Coruña. España ISSN 2386-8333

\begin{tabular}{|c|c|c|c|c|c|}
\hline $\begin{array}{l}\text { B18 Dificultades de } \\
\text { transporte }\end{array}$ & $0,7 \pm 1,1$ & $0,7 \pm 1,2$ & $0,5 \pm 1,0$ & $0,9 \pm 1,2$ & $0,7 \pm 1,2$ \\
\hline $\begin{array}{l}\text { B19 No tengo con quien } \\
\text { hacer ejercicio }\end{array}$ & $0,3 \pm 0,6$ & $0,0 \pm 0,0$ & $0,4 \pm 0,7$ & $0,2 \pm 0,4$ & $0,0 \pm 0,0$ \\
\hline $\begin{array}{l}\text { B20 Tengo que ocuparme } \\
\text { de las tareas del hogar }\end{array}$ & $0,7 \pm 0,9$ & $0,1 \pm 0,3$ & $0,4 \pm 0,7$ & $0,7 \pm 1,0$ & $0,0 \pm 0,0$ \\
\hline $\begin{array}{l}\text { B21 Tengo mal estado de } \\
\text { salud }\end{array}$ & $0,1 \pm 0,2$ & $0,6 \pm 1,2$ & $0,2 \pm 0,4$ & $0,5 \pm 1,0$ & $0,0 \pm 0,0$ \\
\hline $\begin{array}{l}\text { B22 Horarios } \\
\text { incompatibles }\end{array}$ & $0,6 \pm 0,8$ & $1,0 \pm 0,9$ & $0,6 \pm 0,9$ & $0,7 \pm 0,8$ & $0,7 \pm 1,2$ \\
\hline B23 No tengo costumbre & $0,2 \pm 0,4$ & $0,0 \pm 0,0$ & $0,1 \pm 0,3$ & $0,2 \pm 0,4$ & $0,3 \pm 0,6$ \\
\hline $\begin{array}{l}\text { B24 No se oferta la } \\
\text { actividad que me gusta }\end{array}$ & $0,1 \pm 0,3$ & $0,0 \pm 0,0$ & $0,2 \pm 0,4$ & $0,0 \pm 0,0$ & $0,0 \pm 0,0$ \\
\hline $\begin{array}{l}\text { B25 No veo necesario } \\
\text { hacer ejercicio }\end{array}$ & $0,0 \pm 0,0$ & $0,0 \pm 0,0$ & $0,0 \pm 0,0$ & $0,0 \pm 0,0$ & $0,0 \pm 0,0$ \\
\hline $\begin{array}{l}\text { B26 Tengo que cuidar a } \\
\text { familiares mayores }\end{array}$ & $0,0 \pm 0,0$ & $0,1 \pm 0,3$ & $0,0 \pm 0,0$ & $0,1 \pm 0,3$ & $0,0 \pm 0,0$ \\
\hline $\begin{array}{l}\text { B27 Considero mi } \\
\text { discapacidad una barrera }\end{array}$ & $0,2 \pm 0,5$ & $0,6 \pm 1,1$ & $0,3 \pm 0,6$ & $0,5 \pm 1,0$ & $0,3 \pm 0,6$ \\
\hline $\begin{array}{l}\text { B28 No existe una amplia } \\
\text { oferta de AFs adaptadas }\end{array}$ & $1,1 \pm 1,2$ & $1,1 \pm 1,1$ & $0,9 \pm 1,0$ & $1,6 \pm 1,3$ & $0,3 \pm 0,6$ \\
\hline
\end{tabular}

$\mathrm{D}=$ Discapacidad. $* p<0.05$ diferencias significativas con respecto grupo de discapacidad adquirida.

\# $p<0.05$ diferencias significativas con respecto al grupo "4-8 años de experiencia" $\mathrm{y}$ " $>9$ años de experiencia".

\section{Discusión}

El objetivo principal de este estudio fue analizar los motivos y las barreras para la práctica de AF percibidas por personas con DV. La principal contribución del presente estudio ha sido el análisis de los motivos y barreras en participantes de goalball, una modalidad deportiva exclusiva para deportistas con DV, ya que la mayoría de trabajos existentes se han realizado con personas sedentarias o con personas con DV no deportistas. Por otro lado, en el presente estudio también se ha evaluado si existían diferencias en los motivos y barreras percibidas según el sexo, el origen de la discapacidad y los años de experiencia en la práctica de otras modalidades deportivas previas a la práctica del goalball. Salvo en el ítem M12 "Para eliminar el estrés", no se encontraron diferencias entre mujeres y hombres en los motivos ni

Para citar este artículo utilice la siguiente referencia: Avalos Solitario, A.B.; Iturricastillo Urteaga, A.; Castillo Alvira, D.; Romaratezabala Aldasoro, E.; Rodríguez Negro, J.; Aritzeta de Pérdigo, I.; Yanci Irigoyen, J. (2021). Motivos y barreras para la práctica de actividad física percibidas por jugadores de goalball con discapacidad visual. Sportis Sci J, 7 (1), 43-66. https://doi.org/10.17979/sportis.2021.7.1.6713 
Artículo Original. Motivos y barreras para la práctica de actividad física percibidas por jugadores de goalball con discapacidad visual. Vol. 7, n. ${ }^{\circ}$; p. 43-66, enero 2021. A Coruña. España ISSN 2386-8333

en las barreras percibidas. En relación al origen de la discapacidad, únicamente se encontraron diferencias significativas en el ítem B2 "Me da vergüenza que me vean haciendo ejercicio", entre deportistas con discapacidad congénita y adquirida, siendo las personas con discapacidad adquirida quienes más vergüenza sufrían. Con respecto a la comparativa en función de los años de experiencia, salvo en el ítem M17"Porque me gusta superar retos" no se encontraron diferencias significativas entre los diferentes grupos (discapacidad congénita y adquirida). Los resultados del presente estudio pueden ser relevantes para conocer cuáles son los motivos y las barreras percibidas por las personas con DV que practican goalball y, en consecuencia, poder orientar tanto la práctica de la actividad del goalball, como cualquier otra AF dirigida a estas personas con DV.

Debido a la diversidad existente entre las diferentes discapacidades, puede resultar necesario conocer los motivos que perciben las personas con distintas discapacidades, para poder desarrollar una práctica de AF de mayor calidad y más orientada a las necesidades de los practicantes. Teniendo en cuenta la importancia que puede tener conocer los motivos y las barreras percibidas, en la literatura científica este tema ha sido estudiado anteriormente en distintos colectivos de personas con discapacidad (Ramírez, Sanz y Figueroa, 2007; Torralba, Braz y Rubio, 2017; Muñoz, Garrote y Sánchez, 2017; Mendia, Iturricastillo, RodríguezNegro, Romarate y Yanci, 2020) y más concretamente en personas con DV (Jaarsma, Dekker, Koopmans, Dijkstra y Geertzen, 2014; Phoenix, Giffin y Smith, 2015; Haegele, Zhu, Lee y Lieberman, 2016). Los resultados obtenidos en el presente estudio muestran que los motivos más relevantes para la práctica del goalball fueron M19 "Por las sensaciones positivas que me produce practicar AF", "Porque me gusta practicar esta actividad física", M7 "Por diversión" y M8 "Porque disfruto aprendiendo cosas nuevas". Un total de 16 ítems obtuvieron 2 puntos o más (escala 0-3), entre los que encontramos también los ítems M14 "Para relacionarme con otras personas" y M10 "Para mejorar mi bienestar mental". Por el contrario, el ítem M26 "Para compartir actividades con mis hij@s", M1 "Para recuperarme de una enfermedad o lesión", M15 "Para pasar tiempo con mi pareja” y M22 "Por recomendación médica”, fueron los ítems que menos puntuación recibieron $(<0,5$ puntos, escala $0-3)$. Estos resultados coinciden con aquellos obtenidos en investigaciones similares. Concretamente en un estudio

Para citar este artículo utilice la siguiente referencia: Avalos Solitario, A.B.; Iturricastillo Urteaga, A.; Castillo Alvira, D.; Romaratezabala Aldasoro, E.; Rodríguez Negro, J.; Aritzeta de Pérdigo, I.; Yanci Irigoyen, J. (2021). Motivos y barreras para la práctica de actividad física percibidas por jugadores de goalball con discapacidad visual. Sportis Sci J, 7 (1), 43-66. https://doi.org/10.17979/sportis.2021.7.1.6713 
Artículo Original. Motivos y barreras para la práctica de actividad física percibidas por jugadores de goalball con discapacidad visual. Vol. 7, n. $^{\circ}$; p. 43-66, enero 2021. A Coruña. España ISSN 2386-8333

realizado por Muñoz et al. (2017), donde se evaluaron los motivos para la práctica de AF en un grupo de personas con diferentes discapacidades, obtuvieron valores muy similares, destacando los ítems que hacían referencia tanto al disfrute como a la felicidad que suponía la práctica de AF. En la misma línea, Torralba et al. (2017), en un estudio donde se evaluaron los motivos para la práctica de AF en deportistas con DV y DF, también expusieron que uno de los motivos más relevantes era la diversión. Por su parte, Mendia et al. (2020) en un estudio realizado con deportistas con DI y con PC que jugaban en la Liga Genuine, determinaron que los motivos con mayor puntuación fueron los relacionados con el gusto por la AF practicada, para mejorar el bienestar mental y nuevamente, por diversión. En diferentes estudios realizados en personas con $\mathrm{DV}$, se expone que la diversión al realizar la práctica de $\mathrm{AF}$ (Ramírez, Sanz y Figueroa, 2007), la mejora de la salud y el contacto social (Jaarsma, Dekker, Koopmans, Dijkstra y Geertzen, 2014) fueron los motivos más destacados. Jaarsma et al. (2014), además, expusieron que los motivos menos relevantes para la práctica de AF fueron los relacionados con la recomendación médica. Teniendo en cuenta los motivos declarados en la mayor parte de las investigaciones y también en el presente estudio en deportistas con DV, podría ser recomendable que las AF dirigidas a personas con DV tuvieran un importante componente lúdico, donde se prime la práctica grupal y las actividades propuestas sean novedosas y desafiantes. Es más, podría ser recomendable ofertar actividades encaminadas a conseguir un impacto positivo en la salud y el bienestar general.

De la misma forma que los motivos para la práctica de $\mathrm{AF}$, las barreras percibidas por personas con discapacidad también han sido analizadas en trabajos anteriores (Lieberman, Houston-Wilson y Kozub, 2002; Rimmer, Riley, Wang, Rauworth y Jurkowski, 2004; Abellán y Januário, 2017; Lazcano, Ortuza y Romero, 2017; Muñoz, Garrote y Sánchez, 2017). Los resultados obtenidos en el presente estudio sobre las barreras percibidas por deportistas con DV, han tenido unos valores muy bajos, sin superar en ningún caso el valor de 1,2 puntos (escala 0-3). Los valores más altos obtenidos han sido en los ítems B10 "No hay espacios próximos donde practicar" $(1,2 \pm 1,3)$, B28 "No existe una amplia oferta de actividades físicas adaptadas" $(1,2 \pm 1,2)$ y B18 "Dificultades de transporte" $(0,9 \pm 1,1)$. Por otro lado, los ítems B16"No me siento capaz" y "B25 No veo necesario hacer ejercicio", tuvieron una

Para citar este artículo utilice la siguiente referencia: Avalos Solitario, A.B.; Iturricastillo Urteaga, A.; Castillo Alvira, D.; Romaratezabala Aldasoro, E.; Rodríguez Negro, J.; Aritzeta de Pérdigo, I.; Yanci Irigoyen, J. (2021). Motivos y barreras para la práctica de actividad física percibidas por jugadores de goalball con discapacidad visual. Sportis Sci J, 7 (1), 43-66. https://doi.org/10.17979/sportis.2021.7.1.6713 


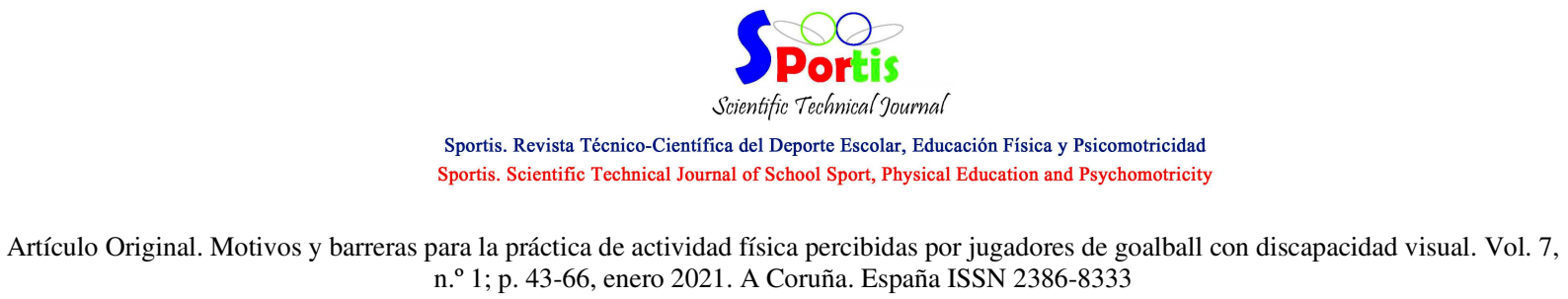

puntuación de 0 puntos. Los resultados obtenidos en el presente estudio son similares a los obtenidos en estudios anteriores. Muñoz et al. (2017), en un estudio realizado con personas con diferentes discapacidades practicantes de AF, obtuvieron que las barreras percibidas fueron, entre otras, la falta de instalaciones, el coste económico y la prestación insuficiente de servicios. Lazcano et al. (2017), en un estudio realizado con personas discapacitadas de la comunidad autónoma del País Vasco, de una edad comprendida entre los 16 y los 29 años, determinaron que la propia discapacidad y el transporte eran una barrera para la práctica de la AF. En un estudio reciente (Mendia et al., 2020), se observó que las barreras menos puntuados fueron B16 "No me siento capaz" y B8 "Los personas que dirigen la actividad no son adecuados". Concretamente en personas con DV, Jaarsma et al. (2014) mostraron como barreras las relacionadas con el transporte, la falta de ofertas cerca del lugar de residencia y la dependencia de otros para la práctica de AF eran percibidas como muy relevantes. De forma similar, Phoenix et al. (2015), en un estudio realizado con personas mayores con DV, obtuvieron que las barreras más relevantes eran los malos horarios de las AF para las personas con discapacidad, las malas instalaciones, el servicio de transporte y la falta de información sobre la oferta de AF. A pesar de que los resultados obtenidos en el presente estudio coinciden con la mayor parte de estudios realizados anteriormente, cabe resaltar que los deportistas con DV participantes en el estudio declararon valores muy cercanos a 0 en muchas de las barreras. La baja percepción en las barreras obtenida en este estudio puede ser debido a las características de la muestra, personas con DV ya practicantes de una modalidad deportiva, y pone de manifiesto que muchas de las barreras percibidas por personas sedentarias o no deportistas con discapacidad ya han sido superadas por los deportistas con DV participantes en el estudio. Aun así, las barreras con mayor puntuación obtenidas por los deportistas con DV coinciden con las barreras declaradas en otros estudios (Jaarsma, Dekker, Koopmans, Dijkstra y Geertzen, 2014; Phoenix, Giffin y Smith, 2015) y también con las barreras más relevantes a modificar en las estrategias de diferentes planes de acción planteados por distintas instituciones (Comisión Europea, 2010, Ministerio de Sanidad, Política e Igualdad de España, 2014). Por ejemplo, desde la Comisión Europea (2010) se expusieron diferentes estrategias para mejorar la práctica de AF de personas con discapacidad, como por ejemplo

Para citar este artículo utilice la siguiente referencia: Avalos Solitario, A.B.; Iturricastillo Urteaga, A.; Castillo Alvira, D.; Romaratezabala Aldasoro, E.; Rodríguez Negro, J.; Aritzeta de Pérdigo, I.; Yanci Irigoyen, J. (2021). Motivos y barreras para la práctica de actividad física percibidas por jugadores de goalball con discapacidad visual. Sportis Sci J, 7 (1), 43-66. https://doi.org/10.17979/sportis.2021.7.1.6713 
Artículo Original. Motivos y barreras para la práctica de actividad física percibidas por jugadores de goalball con discapacidad visual. Vol. 7, n. $^{\circ}$; p. 43-66, enero 2021. A Coruña. España ISSN 2386-8333

mejorar la accesibilidad en todos los ámbitos y la organización de actos específicos para las personas con discapacidad. Por otro lado, desde el Ministerio de Sanidad, Política e Igualdad de España (2014) y al hilo de lo establecido por la Comisión Europea, se plantearon diferentes líneas estratégicas, como el promover la sensibilización sobre la diversidad de la discapacidad en el sector del transporte. En un estudio reciente realizado en la comunidad de Madrid sobre la población con discapacidad (Pinilla y Pérez-Tejero, 2017), se estableció que se debía favorecer la movilidad y aumentar la oferta de actividades físicas adaptadas. Teniendo en cuenta que los deportistas con DV participantes en el presente estudio, a pesar de tener puntuaciones muy bajas en la mayor parte de ítems referentes a las barreras posiblemente por ser ya practicantes y haber superado muchas de ellas, también perciben estas barreras, podía ser interesante focalizar los esfuerzos en mejorar los espacios de práctica de AF de forma generalizada, la oferta de actividades físicas adaptadas y el transporte para personas con discapacidad.

Una de las principales aportaciones del presente estudio es el análisis de los motivos y barreras percibidas por personas con discapacidad atendiendo al sexo, al origen de la discapacidad y a la experiencia en la práctica deportiva. Los resultados obtenidos muestran que el único ítem en el que se observaron diferencias significativas entre hombres y mujeres fue M12 "Para eliminar el estrés", declarando las mujeres valores más altos que los hombres $(2,8 \pm 0,6$ vs. $1,8 \pm 1,0, p<0,05)$. Con respecto al origen de la discapacidad, únicamente en el ítem B2 "Me da vergüenza que me vean haciendo ejercicio" los deportistas con discapacidad congénita declararon valores significativamente más altos que los deportistas con discapacidad adquirida $(p<0,05)$. En el caso de la experiencia deportiva, se observaron diferencias significativas entre el grupo de "4-8 años de experiencia" y el grupo de ">9 años de experiencia" únicamente en el ítemM17 "Porque me gusta superar retos". En el caso de las barreras no se percibió ninguna diferencia significativa ni en función del sexo, ni del origen de la discapacidad ni de los años de experiencia. En el ámbito de la discapacidad, no hemos encontrado trabajos que traten dichas comparativas en función del sexo, origen de la discapacidad o la experiencia en el deporte. Sin embargo, en un trabajo sobre personas adultas sin discapacidad de la comunidad de Madrid (Kostiuk, Rodriguez y Barripedro, 2016), se

Para citar este artículo utilice la siguiente referencia: Avalos Solitario, A.B.; Iturricastillo Urteaga, A.; Castillo Alvira, D.; Romaratezabala Aldasoro, E.; Rodríguez Negro, J.; Aritzeta de Pérdigo, I.; Yanci Irigoyen, J. (2021). Motivos y barreras para la práctica de actividad física percibidas por jugadores de goalball con discapacidad visual. Sportis Sci J, 7 (1), 43-66. https://doi.org/10.17979/sportis.2021.7.1.6713 


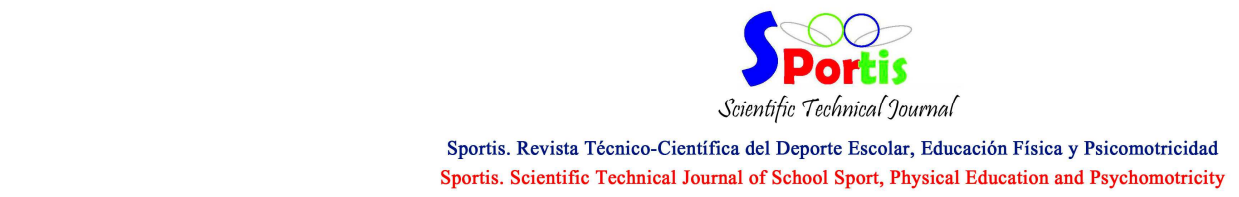

Artículo Original. Motivos y barreras para la práctica de actividad física percibidas por jugadores de goalball con discapacidad visual. Vol. 7 , n. $^{\circ}$; p. 43-66, enero 2021. A Coruña. España ISSN 2386-8333

evaluaron las barreras percibidas en la práctica de AF y deporte en función del sexo. Las mujeres presentaron valores más altos en las barreras B6 "estoy cansado", B11 "no me gusta", B20 "tengo que ocuparme de tareas del hogar" y B7 "es demasiado caro" con respecto a los hombres. En otro artículo llevado a cabo con estudiantes universitarios (Sevil, Praxedes, Zaragoza, Villar y García-González, 2017), se muestra que las barreras referidas al tiempo y a la capacidad de disfrute obtuvieron mayor puntuación en las mujeres. Las escasas diferencias observadas tanto en los motivos como en las barreras percibidas por los participantes con DV del presente estudio en función del sexo, el origen de la discapacidad o la experiencia en el deporte pueden ser debidas a que todos eran deportistas de contextos similares (goalball). Posiblemente la práctica común de una actividad deportiva pueda minimizar las diferencias sobre los motivos y barreras para la práctica de AF.

El presente estudio no está exento de limitaciones. Cabe destacar que la muestra (tamaño y características) no es representativa de las personas con discapacidad, ni tan siquiera de los deportistas con DV. Sería interesante en futuras investigaciones realizar estudios con muestras mayores y con potencia estadística suficiente como para poder hacer generalizaciones al respecto. Además, todos los deportistas participantes fueron reclutados del mismo entorno deportivo, en este caso el goalball. Por todo ello, podría ser interesante llevar a cabo otros estudios similares en distintas modalidades deportivas practicadas por personas con DV. La presente investigación puede ser una aproximación inicial para conocer los motivos y barreras percibidas por deportistas con DV pero resultarían necesarios más estudios con muestras más diversas con el fin de poder obtener conclusiones relevantes.

\section{Conclusión}

Los resultados obtenidos en el presente estudio muestran que los motivos para la práctica de AF más valorados por los deportistas participantes fueron los relacionados con la diversión y con la necesidad de que produzcan sensaciones positivas. En este sentido, sería interesante que las actividades dirigidas a personas con DV pudieran tener presentes estos dos componentes. Por otro lado, las barreras más valoradas, aunque la mayor parte de ellas obtuvieron una baja puntuación, fueron "No hay espacios próximos donde practicar", "No

Para citar este artículo utilice la siguiente referencia: Avalos Solitario, A.B.; Iturricastillo Urteaga, A.; Castillo Alvira, D.; Romaratezabala Aldasoro,

E.; Rodríguez Negro, J.; Aritzeta de Pérdigo, I.; Yanci Irigoyen, J. (2021). Motivos y barreras para la práctica de actividad física percibidas por jugadores de goalball con discapacidad visual. Sportis Sci J, 7 (1), 43-66. https://doi.org/10.17979/sportis.2021.7.1.6713 
Artículo Original. Motivos y barreras para la práctica de actividad física percibidas por jugadores de goalball con discapacidad visual. Vol. 7 , n. $^{\circ}$; p. 43-66, enero 2021. A Coruña. España ISSN 2386-8333

existe una amplia oferta de actividades físicas adaptadas" y "Dificultades de transporte". La baja puntuación obtenida en la mayor parte de las barreras puede ser debido a que los participantes en el estudio eran deportistas con una experiencia alta y practicantes de AF de forma asidua, pudiendo estar superadas muchas de las barreras presentadas. Cabe destacar que pese a que las puntuaciones obtenidas en las barreras han sido bajas, las más puntuadas concuerdan con las barreras percibidas en otros estudios.

Por otro lado, salvo en algunos ítems concretos, no se encontraron diferencias en los motivos y las barreras atendiendo al sexo, al origen de la discapacidad y a los años de experiencia previa en otras disciplinas deportivas. Este aspecto pone de manifiesto que, quizá, la práctica de una modalidad deportiva como el goalball pueda favorecer la eliminación de diferencias en función del sexo, del tipo de discapacidad o de la experiencia deportiva en cuanto a los motivos y las barreras percibidas para la práctica de AF.
\end{abstract}

\title{
Referencias
}

Abellán, J., y Januário, N. (2017). Barreras, facilitadores y motivos de la práctica deportiva de deportistas con discapacidad intelectual. Psychology, Society and Education, 9(3), 419-431. DOI: https://doi.org/10.25115/psye.v9i3.1023

Aviram, R., Harries, N., Rabani, A. S., Amro, A., Nammourah, I., Al-Jarrah, M., Raanan, Y., Hutzler, Y., y Bar-Haim, S. (2019). Comparison of habitual physical activity and sedentary behavior in adolescents and young adults with and without cerebral palsy. Pediatric Exercise Science, 31(1), 60-66. DOI: https://doi.org/10.1123/pes.2017-0285

Bonet, J., Parrado, E., y Capdevila, L. (2017). Efectos agudos del ejercicio físico sobre el estado de ánimo y la HRV. Revista Internacional de Medicina y Ciencias de la Actividad Física y el Deporte, 17(65), 85-100. DOI: http://dx.doi.org/10.15366/rimcafd2017.65.006

Brizuela Costa, G., Romero Ávila, J. L., y Beltrán Herranz, J. (2016). Lesión medular y ejercicio físico: revisión desde una perspectiva deportiva. Revista Española De Discapacidad, 4(2), 163-185. DOI: https://doi.org/10.5569/2340-5104.04.02.09

Çıtak, I., Aki, E., y Ergun, N. (2009). Physical Fitness of Visually Impaired Adolescent Goalball Players. Perceptual and Motor Skills, 108, 129-136. DOI: https://doi.org/10.2466/pms.108.1.129-136

Çolak, T., Bamaç, B., Aydin, M., Meriç, B., y Özbek, A. (2004). Physical fitness levels of blind and visually impaired goalball team players. Isokinetics and Exercise Science,

Para citar este artículo utilice la siguiente referencia: Avalos Solitario, A.B.; Iturricastillo Urteaga, A.; Castillo Alvira, D.; Romaratezabala Aldasoro,

E.; Rodríguez Negro, J.; Aritzeta de Pérdigo, I.; Yanci Irigoyen, J. (2021). Motivos y barreras para la práctica de actividad física percibidas por jugadores de goalball con discapacidad visual. Sportis Sci J, 7 (1), 43-66. https://doi.org/10.17979/sportis.2021.7.1.6713 
Artículo Original. Motivos y barreras para la práctica de actividad física percibidas por jugadores de goalball con discapacidad visual. Vol. 7 , n. ${ }^{\circ}$ 1; p. 43-66, enero 2021. A Coruña. España ISSN 2386-8333

12(4), 247-252. DOI: https://doi.org/10.3233/ies-2004-0182

Comisión Europea (2010). Estrategia Europea sobre Discapacidad 2010-2020: un compromiso renovado para una Europa sin barreras. Retrieved from https://www.mscbs.gob.es/ssi/discapacidad/docs/estrategia_europea_discapacidad_201 0_2020.pdf

Conchar, L., Bantjes, J., Swartz, L., y Derman, W. (2016). Barriers and facilitators to participation in physical activity: The experiences of a group of South African adolescents with cerebral palsy. Journal of Health Psychology, 21(2), 152-163. DOI: https://doi.org/10.1177/1359105314523305

Copado, C. A., Palomar, V. G., Ureña, A. M., Mengual, F. A., Martínez, M. S., y Serralta, J. R. L. (2011). Mejora en el control de los diabéticos tipo 2 tras una intervención conjunta: educación diabetológica y ejercicio físico. Atención primaria, 43(8), 398-406.

Dairo, Y. M., Collett, J., Dawes, H., y Oskrochi, G. R. (2016). Physical activity levels in adults with intellectual disabilities: A systematic review. Preventive Medicine Reports, 4, 209-219. DOI: https://doi.org/10.1016/j.pmedr.2016.06.008

Gallego, J., Aguilar-Parra, J. M., Cangas, J. A., Pérez-Escobar, J. M., y Barrera, S. (2014). Hábitos De Actividad Física En Mujeres Con Discapacidad: Relación Con Sus Características Físicas Y Funcionales. Revista Iberoamericana de Psicología del Ejercicio y el Deporte, 9 (2), 471-494.

Garber, C. E., Blissmer, B., Deschenes, M. R., Franklin, B. A., Lamonte, M. J., Lee, I. M., Nieman, D. C., y Swain, D. P. (2011). Quantity and quality of exercise for developing and maintaining cardiorespiratory, musculoskeletal, and neuromotor fitness in apparently healthy adults: Guidance for prescribing exercise. Medicine and Science in Sports and Exercise, 43(7), 1334-1359. DOI: https://doi.org/10.1249/MSS.0b013e318213fefb

Haegele, J. A., Zhu, X., Lee, J., y Lieberman, L. J. (2016). Physical activity for adults with visual impairments: Impact of socio-demographic factors. European Journal of Adapted Physical Activity, 9(1), 3-14. DOI: https://doi.org/10.5507/euj.2016.001

Heras, N., Reina, R., Roldán, A., Iturricastillo, A., Rodríguez-Negro, J., y Yanci, J. (2019). Efectos de la actividad física en las capacidades funcionales de personas con parálisis cerebral : una revisión sistemática. Revista Española de Educación Física y Deportes, 427, 55-69.

Houston-Wilson, C., y Kozub, F. M. (2002). Perceived barriers to including students with visual impairments in general physical education. Adapted Physical Activity Quarterly, 19(3), 364-377. DOI: https://doi.org/10.1123/apaq.19.3.364

Jaarsma, E. A., Geertzen, J. H. B., de Jong, R., Dijkstra, P. U. y Dekker, R. (2014). Barriers and facilitators of sports in Dutch Paralympic athletes: An explorative study.

Para citar este artículo utilice la siguiente referencia: Avalos Solitario, A.B.; Iturricastillo Urteaga, A.; Castillo Alvira, D.; Romaratezabala Aldasoro,

E.; Rodríguez Negro, J.; Aritzeta de Pérdigo, I.; Yanci Irigoyen, J. (2021). Motivos y barreras para la práctica de actividad física percibidas por jugadores de goalball con discapacidad visual. Sportis Sci J, 7 (1), 43-66. https://doi.org/10.17979/sportis.2021.7.1.6713 
Artículo Original. Motivos y barreras para la práctica de actividad física percibidas por jugadores de goalball con discapacidad visual. Vol. 7 , n. ${ }^{\circ} 1$; p. 43-66, enero 2021. A Coruña. España ISSN 2386-8333

Scandinavian Journal of Medicine and Science in Sports, 24(5), 830-836. DOI: https://doi.org/10.1111/sms.12071

Kostiuk, L. V. (2016). Barreras para la práctica de actividad física y deportiva en las personas adultas de la Comunidad de Madrid. Desarrollo y validación de un instrumento. DOI: https://doi.org/10.20868/UPM.thesis.39693

Lazcano, I., Ortuzar, A., y Romero, S. (2017). Determinantes de las barreras percibidas entre los jóvenes con discapacidad para no participar en prácticas deportivas. Rivista Italiana Di Pedagogia Dello Sport, 1, 52-60. DOI: https://doi.org/10.5281/zenodo.1065499

Lieberman, L. J., Houston-Wilson, C., y Kozub, F. M. (2002). Perceived barriers to including students with visual impairments in general physical education. Adapted Physical Activity Quarterly, 19(3), 364-377. DOI: https://doi.org/10.1123/apaq.19.3.364

McKeon, M., Slevin, E., y Taggart, L. (2013). A pilot survey of physical activity in men with an intellectual disability. Journal of Intellectual Disabilities, 17(2), 157-167. DOI: https://doi.org/10.1177/1744629513484666

Mendia, U., Iturricastillo, A., Rodríguez-Negro, J., Romarate, A. y Yanci, J. (2020). Percepción de las barreras y facilitadores para la práctica de actividad física de futbolistas de la Liga Genuine. Revista Iberoamericana de Ciencias de la Actividad $\begin{array}{lllll}\text { Física } y & \text { el } & \text { Deporte } & \text { 9(1), 95-105. DOI: }\end{array}$ https://doi.org/10.24310/riccafd.2020.v9i1.8304

Montalvo, D., y Jalomo, M. K. (2016). Intervención cognitiva en el adulto mayor con discapacidad. Trabajo Recepcional de Titulación para obtener el grado de Maestría en Salud Pública. Universidad Autónoma de Nayarit.

Muñoz Jiménez, E. M., Garrote Rojas, D., y Sánchez Romero, C. (2017). La práctica deportiva en personas con discapacidad: motivación personal, inclusión y salud. International Journal of Developmental and Educational Psychology. Revista INFAD de Psicología., 4(1), 145. DOI: https://doi.org/10.17060/ijodaep.2017.n1.v4.1037

Organización Mundial de la Salud. (2019). Actividad física. Disponible en: https://www.who.int/es/news-room/fact-sheets/detail/physical-activity

Petro, A., Herazo, Y., y Pinillos, Y. (2019). Niveles de actividad física en personas con discapacidad de la región caribe y nororiente de Colombia. Proyecto de investigación para obtener el título de Magister en Actividad Física y salud. Universidad Simón Bolívar. Colombia.

Pinilla Arbex, J., y Pérez-Tejero, J. (2017). Situación actual de la actividad física para personas con discapacidad en la Comunidad de Madrid. Revista Española de Discapacidad, 5(1), 153-165. DOI: https://doi.org/10.5569/2340-5104.05.01.09

Place, K. y Hodge, S. R. (2001). Social inclusion of students with physical disabilities in general physical education: A behavioral analysis. Adapted Physical Activity Quarterly

Para citar este artículo utilice la siguiente referencia: Avalos Solitario, A.B.; Iturricastillo Urteaga, A.; Castillo Alvira, D.; Romaratezabala Aldasoro, E.; Rodríguez Negro, J.; Aritzeta de Pérdigo, I.; Yanci Irigoyen, J. (2021). Motivos y barreras para la práctica de actividad física percibidas por jugadores de goalball con discapacidad visual. Sportis Sci J, 7 (1), 43-66. https://doi.org/10.17979/sportis.2021.7.1.6713 
Artículo Original. Motivos y barreras para la práctica de actividad física percibidas por jugadores de goalball con discapacidad visual. Vol. 7 , n. ${ }^{\circ} 1$; p. 43-66, enero 2021. A Coruña. España ISSN 2386-8333

18, 389-404. DOI: https://doi.org/10.1123/apaq.18.4.389

Phoenix, C., Griffin, M., y Smith, B. (2015). Physical activity among older people with sight loss: A qualitative research study to inform policy and practice. Public Health, 129(2), 124-130. DOI: https://doi.org/10.1016/j.puhe.2014.10.001

Ramirez, A., Sanz, S. y Figueroa de la Paz, A. (2007). Estudio sobre los hábitos deportivos en las personas con discapacidad en la provincia de Guipuzcoa. Federación Guipuzcoana de Deporte Adaptado.

Rimmer, J. H., Riley, B., Wang, E., Rauworth, A., y Jurkowski, J. (2004). Physical activity participation among persons with disabilities: Barriers and facilitators. American Journal of Preventive Medicine, 26(5), 419-425. DOI: https://doi.org/10.1016/j.amepre.2004.02.002

Sadowska, D., y Krzepota, J. (2015). Assessment of physical activity of people with visual impairments and individuals who are sighted using the international physical activity questionnaire and actigraph. Journal of Visual Impairment and Blindness, 109(2), 119-129. DOI: https://doi.org/10.1177/0145482x1510900207

Sevil, J., Praxedes, A., Zaragoza, J., Villar, F., y Garcia-Gonzalez, 1. (2017). Barreras percibidas para la práctica de actividad física en estudiantes universitarios. Diferencias por género y niveles de actividad física. Universitas Psychologica. 16(4), 303-317. DOI: https://doi.org/10.11144/Javeriana.upsy16-4.bppa

Smith, L., Jackson, S. E., Pardhan, S., López-Sánchez, G. F., Hu, L., Cao, C., Vancampfort, D., Koyanagi, A., Stubbs, B., Firth, J., y Yang, L. (2019). Visual impairment and objectively measured physical activity and sedentary behaviour in US adolescents and adults: A cross-sectional study. BMJ Open, 9(4). DOI: https://doi.org/10.1136/bmjopen-2018-027267

Smith, B., Kirby, N., Skinner, B., Wightman, L., Lucas, R., y Foster, C. (2019). Infographic. Physical activity for disabled adults. British Journal of Sports Medicine, 53(6), 335336. DOI: https://doi.org/10.1136/bjsports-2018-100158

Ministerio de Sanidad. (2014). Plan de acción de Estrategia Española Sobre Discapacidad 2014-2020. 1-30.

Torralba, M. Á., Braz, M., y Rubio, M. J. (2017). La motivación en el deporte adaptado. Psychology, Society, \& Education, 6(1), 27. DOI: https://doi.org/10.25115/psye.v6i1.506

Verschuren, O., Wiart, L., Hermans, D., y Ketelaar, M. (2012). Identification of facilitators and barriers to physical activity in children and adolescents with cerebral palsy. Journal of Pediatrics, 161(3), 488-494. DOI: https://doi.org/10.1016/j.jpeds.2012.02.042 DOI:

Wilson, M. G., Ellison, G. M., y Cable, N. T. (2016). Basic science behind the cardiovascular benefits of exercise. British Journal of Sports Medicine, 50(2), 93-99.

Para citar este artículo utilice la siguiente referencia: Avalos Solitario, A.B.; Iturricastillo Urteaga, A.; Castillo Alvira, D.; Romaratezabala Aldasoro, E.; Rodríguez Negro, J.; Aritzeta de Pérdigo, I.; Yanci Irigoyen, J. (2021). Motivos y barreras para la práctica de actividad física percibidas por jugadores de goalball con discapacidad visual. Sportis Sci J, 7 (1), 43-66. https://doi.org/10.17979/sportis.2021.7.1.6713 
Artículo Original. Motivos y barreras para la práctica de actividad física percibidas por jugadores de goalball con discapacidad visual. Vol. 7, n. ${ }^{\circ} 1$; p. 43-66, enero 2021. A Coruña. España ISSN 2386-8333

https://doi.org/10.1136/bjsports-2014-306596rep

Wouters, M., Evenhuis, H. M., y Hilgenkamp, T. I. M. (2018). Physical activity levels of children and adolescents with moderate-to-severe intellectual disability. Journal of Applied Research in Intellectual Disabilities, 32(1), 131-142. DOI: https://doi.org/10.1111/jar.12515 